\title{
MARCO TEÓRICO GENERAL PARA EVALUAR LA LECTURA Y DISEÑAR PRÁCTICAS PARA SU ENSEÑANZA
}

\section{General theoretical framework for assessing reading and designing practices for its teaching}

\author{
Ana Belén Domínguez Gutiérrez \\ Universidad de Salamanca \\ Correo-e: abd@usal.es \\ Virginia González Santamaria \\ Universidad de Salamanca \\ Correo-e: virginia_gonzalez_santamaria@usal.es \\ Recibido: 3 de septiembre de 2020 \\ Envío a informantes: I2 de septiembre de 2020 \\ Aceptación definitiva: 18 de octubre de 2020
}

RESUMEN: En este artículo se presenta un marco teórico general para la enseñanza de la lectura o modelo didáctico en el que se expone qué habilidades son necesarias para mejorar la comprensión lectora o, mejor aún, un marco que permita poner en marcha estrategias proactivas, que «no dejen a ningún niño atrás» y que permitan intervenir tempranamente en las dificultades de aprendizaje para evitar que estas lleguen a tener más entidad y sean más resistentes a las acciones educativas. Desde la perspectiva psicolingüística, se identifican los conocimientos y procesos que intervienen en la comprensión lectora y que pueden ser evaluados, enseñados y mejorados, es decir, se propone una guía para diseñar prácticas de enseñanza de la lectura. Se revisan fundamentalmente los denominados aspectos no específicos de la lectura (comunes también a la comprensión de la lengua oral), mostrando por qué son necesarios y cómo evaluarlos. Se analizan las competencias léxicas del lector; los procesos de análisis morfosintáctico y de integración semántica; los conocimientos que posee sobre el mundo, un tema particular o una disciplina; y los conocimientos que tiene sobre las estructuras organizativas de los textos.

Palabras clave: lectura; modelo didáctico lectura; evaluación de la lectura; comprensión lectora. 
AвSTRACT: This article presents a general theoretical framework for the teaching of reading or a didactic model in which it sets out what skills are needed to improve reading comprehension, or better still, a framework that allows proactive strategies to be put in place, which «leave no child behind» and which allow early intervention in learning difficulties to prevent these from becoming more significant and resistant to educational actions. From a psycholinguistic perspective, the knowledge and processes involved in reading comprehension are identified and can be evaluated, taught, and improved, that is, a guide is proposed for designing reading teaching practices. The so-called non-specific aspects of reading (also common to oral language comprehension) are fundamentally reviewed, showing why they are necessary and how to evaluate them. The lexical skills of the reader, the processes of morphosyntactic analysis and semantic integration, the knowledge he or she has about the world, or a particular subject or discipline, and the knowledge he or she has about the organisational structures of texts are analysed.

KEY WORDS: reading; didactic model reading; reading assessment; reading comprehension.

\section{Introducción}

$\mathrm{L}$

A LENGUA ESCRITA es una herramienta esencial para el desarrollo personal y social de las personas pertenecientes a sociedades como la nuestra, donde gran parte de la información es transmitida en su forma escrita. La lengua escrita es un acto de comunicación con unas funciones específicas que llevan a estas personas a interesarse por su aprendizaje. Sin embargo, aprender a leer y escribir es un proceso tremendamente complejo que requiere desarrollar múltiples capacidades de tipo cognitivo, lingüístico y metacognitivo; lo cual provoca que, a pesar de los esfuerzos dedicados a su enseñanza, un considerable número de estudiantes tengan dificultades en su aprendizaje, en tener un progreso lector adecuado y en alcanzar una competencia lectora adecuada y funcional que les permita obtener información y acceder a los conocimientos a través de materiales impresos en papel (libros, manuales, periódicos...) y a través de soportes digitales (correo electrónico, internet, redes sociales...).

Para diseñar y desarrollar estrategias de enseñanza de la lectura es necesario disponer de un marco teórico general sobre la lectura y la escritura que nos permita abordar, por un lado, su enseñanza-aprendizaje y, por otro, conocer las posibles dificultades que puedan surgir en ese proceso. Este es objebivo de este trabajo.

En este marco teórico se asume que la lectura se basa en un código lingüístico que requiere ejecutar procesos cognitivos (apoyados en ciertos conocimientos) y que se usa socialmente en comunidades culturales (fuera de las prácticas socioculturales en las cuales ocurre pierde todo el sentido). Nada de ello es prescindible, por lo que sería mejor combinarlo todo en una práctica educativa integradora que tenga en cuenta el trabajo sobre dos bloques de contenido, uno de ellos inspirado por la perspectiva psicolingüística, basado en los procesos de reconocimiento de las palabra escrita y aspectos no específicos o mecanismos generales de comprensión del lenguaje oral. $\mathrm{Y}$ un segundo bloque inspirado en una perspectiva más comunicativo-multimodal y sociocultural; en los que los objetivos de lectura, es decir, el para qué leer y escribir como instrumento de uso social, marcarán los objetivos de trabajo. Sin embargo, por motivos de espacio este trabajo se centra básicamente en los aspectos psicolingüísticos de la lectura. 
MARCO TEÓRICO GENERAL PARA EVALUAR LA LECTURA

Y DISEÑAR PRÁCTICAS PARA SU ENSEÑANZA

ANA BELÉN DOMÍNGUEZ GUTIÉRREZ Y VIRGINIA GONZÁLEZ SANTAMARÍA

\section{Marco teórico general sobre la lectura}

Con este marco teórico general no se pretende explicar cómo es la comprensión lectora, sino que se trata de presentar una propuesta didáctica en la que se expone qué habilidades son necesarias para desarrollar o mejorar la comprensión lectora o, dicho de otra forma, un marco que permita poner en marcha estrategias proactivas que permitan intervenir tempranamente en las dificultades de aprendizaje para evitar que estas lleguen a tener más entidad y sean más resistentes a las acciones educativas (Oakhill, Cain y Bryant, 2003; Savage, 200I), es decir, pasar de una estrategia reactiva y tardía (esperar al fracaso escolar) a una estrategia proactiva que no deje a ningún niño atrás (Modelo de Respuesta a la Intervención, RTI). Este modelo, centrado en alumnos con riesgo de tener dificultades de aprendizaje, trata de prevenir y anticipar las dificultades de aprendizaje realizando una identificación temprana y una evaluación progresiva de la respuesta del niño (AA. VV., 20I2). Para llevar a la práctica esta propuesta es preciso identificar los conocimientos y procesos que dan lugar a la comprensión lectora y que pueden ser evaluados, enseñados y mejorados, es decir, es una guía para diseñar prácticas de enseñanza de la lengua escrita (Oakhill et al., 2003; Savage, 200I). Asunto que se aborda en este artículo.

El marco teórico que se propone (y diversas teorías sobre la lectura) postula que la comprensión de la lectura es la resultante de dos factores, las habilidades para el reconocimiento de palabras escritas y las habilidades para la comprensión de la lengua oral (Figura I). En consecuencia, las diferencias individuales (incluidas las dificultades) en la comprensión de la lengua escrita pueden deberse a la una (dificultades para recuperar la fonología y la semántica de las palabras a partir de su representación escrita), a la otra (problemas en las capacidades cognitivas y lingüísticas que son necesarias para entender el lenguaje hablado) o las dos (Alegría, 2004; Morais, 1994).

Figura i. Componentes de la comprensión del lenguaje escrito (tomado de Morais, 1994, p. 185).

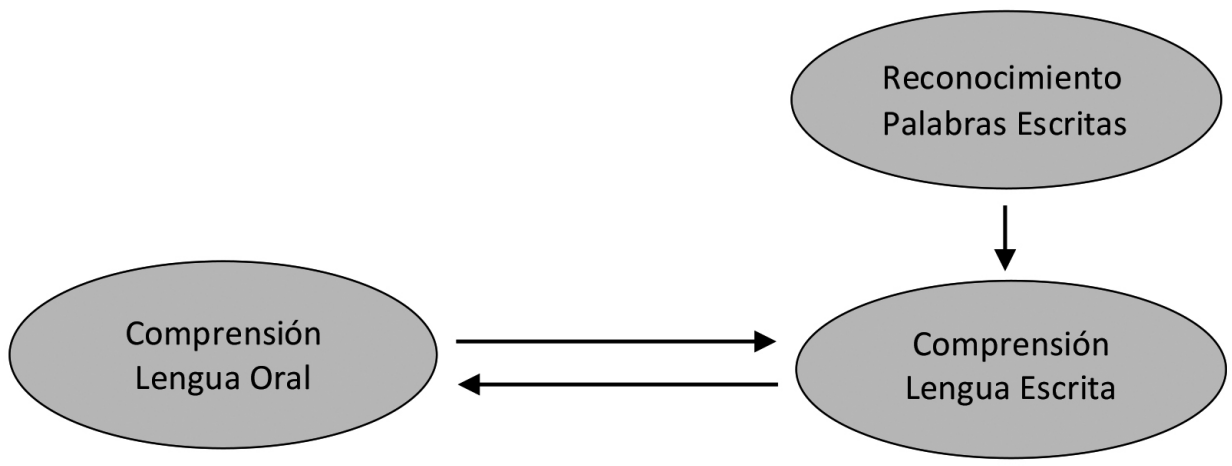

Esta forma de concebir la lectura es lo que Gough y Tunmer (1986) denominaron Modelo Simple de Lectura. Sus autores advierten que el nombre de Simple View of Reading no implica que la lectura sea un proceso «simple», más bien el modelo es una 
forma simple de conceptualizar la complejidad de la lectura, en el sentido de contener muy pocos elementos en su formulación. Será necesario, por eso, identificar las partes constituyentes de cada uno de esos elementos para segmentar el acto de leer (este es el objetivo de este trabajo) y poder diseñar actividades sencillas que, integradas y contextualizadas, permitan mejorar las prácticas de la enseñanza de la lectura, respondiendo así a las prácticas educativas integradoras mencionadas anteriormente.

El marco teórico propuesto en este trabajo (Figura 2) plantea que el primer factor implicado en la comprensión de la lengua escrita tiene que ver con una habilidad no específica de la misma: los conocimientos lingüísticos del lector, esencialmente lexicales, sintácticos y pragmáticos. Para comprender los textos utilizamos nuestras competencias léxicas (es decir, el conocimiento que tenemos del sentido de las palabras), así como procesos de análisis sintáctico y de integración semántica; utilizamos incluso nuestros conocimientos del mundo, nuestra experiencia personal, pero todos estos procesos y conocimientos se ponen también en marcha cuando comprendemos la lengua oral, por eso, se denominan habilidades no específicas de la lectura.

FIGURA 2. Representación gráfica del marco teórico general para interpretar e intervenir en las dificultades de aprendizaje de la lengua escrita.

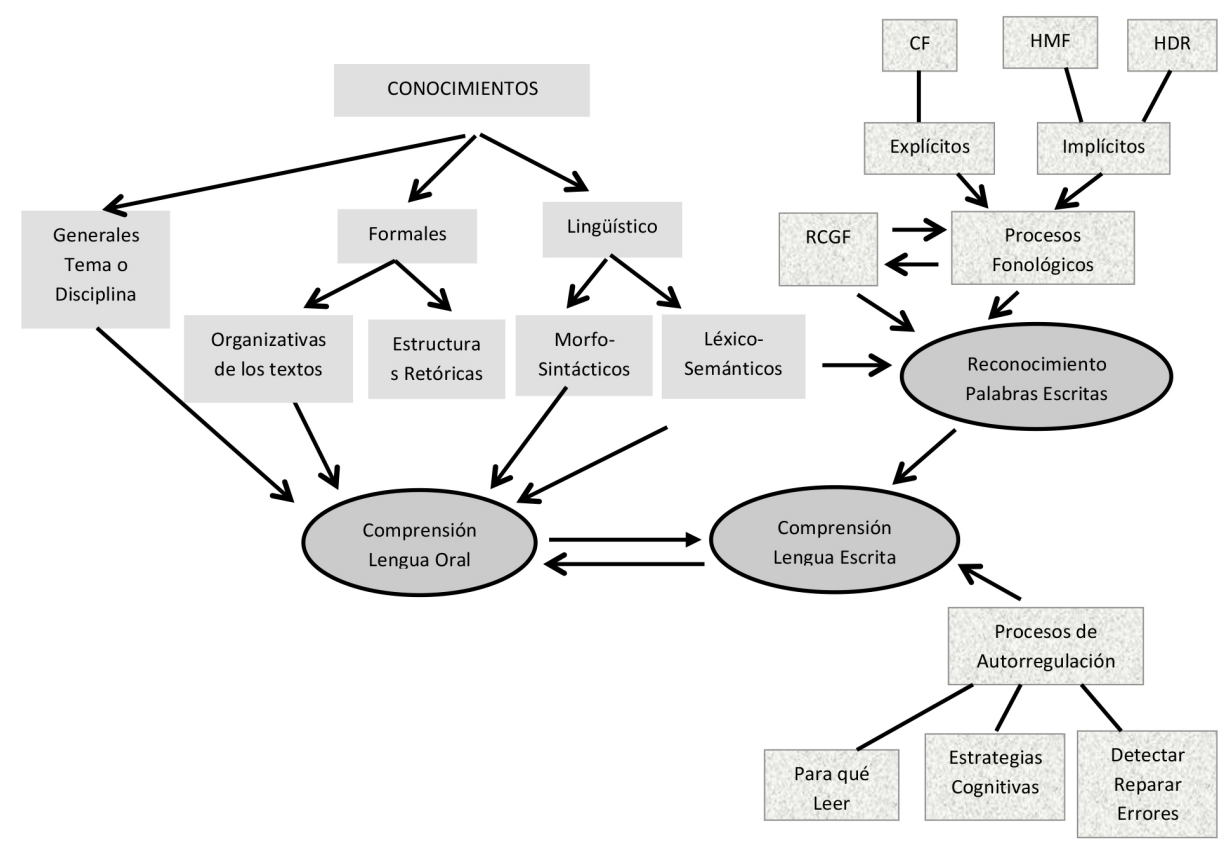

Nota: $\mathrm{CF}=$ Conciencia Fonológica; $\mathrm{HMF}=$ Memoria Fonológica HDR = Habilidades de Denominación Rápida; RCGF = Reglas de conversión Grafema-Fonema).

El segundo factor a considerar dentro de este marco teórico concierne a las habilidades específicas de la lectura. El más importante de todos es el reconocimiento de las palabras escritas. Los mecanismos cognitivos que permiten la identificación de 
MARCO TEÓRICO GENERAL PARA EVALUAR LA LECTURA

Y DISEÑAR PRÁCTICAS PARA SU ENSEÑANZA

ANA BELÉN DOMÍNGUEZ GUTIÉRREZ Y VIRGINIA GONZÁLEZ SANTAMARÍA

palabras escritas hacen intervenir masivamente la fonología (Domínguez y Leybaert, 20I4). Los trabajos experimentales sobre la lectura muestran que cuando se controlan los factores lingüísticos (vocabulario y sintaxis) la fonología aparece como el determinante más importante de las habilidades de lectura (Alegría, 2003; Morais, 1994).

Una de las características más definitorias de un buen lector es su capacidad para leer palabras, reconociendo automáticamente muchas de ellas y descodificando aquellas que le son desconocidas. Estos procesos de reconocimiento de palabras tienen que adquirir un grado importante de automatismo para ser eficaces, es decir, deben poder realizarse al tiempo que la atención del lector se dirige a otro aspecto de la realidad. Si los lectores son capaces de reconocer palabras de forma automática, sin que ello ocupe su atención, podrán entonces dirigir esta hacia la sintaxis y otros aspectos de mayor nivel del lenguaje implicados en la comprensión de un texto, objetivo último de la lectura.

Para ello (véase parte derecha de la Figura 2), junto al empleo de reglas de transformación grafema-fonema, los niños deben aprender las correspondencias entre los sonidos individuales del lenguaje, los fonemas y las letras que representan estos sonidos, los grafemas. Para lo cual, deben centrar su atención sobre los sonidos o acceder a la fonología de su lenguaje, es decir, deben ser capaces de manipular explícitamente los segmentos fonológicos del habla, capacidad que se ha denominado conciencia fonológica (Alegría, 2006; Domínguez, 2009). Esto es, la habilidad de identificar, segmentar o combinar, de forma intencional, las unidades subléxicas de las palabras: sílabas y fonemas. Además, junto a la conciencia fonológica existen otras habilidades fonológicas de carácter implícito que también han mostrado un valor predictivo del aprendizaje de la lectura, aunque han sido menos investigadas (Defior y Serrano, 20II). A diferencia de la conciencia fonológica, que requiere una reflexión explícita sobre los sonidos de la palabra y su manipulación, las habilidades fonológicas de carácter implícito se ponen en marcha automáticamente, es decir, usan información fonológica sin necesidad de reflexionar sobre ella explícitamente. Wagner y Torgesen (1987) señalaron como tales a las habilidades de memoria fonológica o verbal a corto plazo y las de acceso rápido a las representaciones fonológicas almacenadas en la memoria a largo plazo (habilidades de denominación rápida).

Las habilidades específicas de la lectura o reconocimiento de las palabras escritas han sido una de las cuestiones más investigadas en el campo de la lectura, determinando cuáles son los mecanismos que emplea un lector competente para realizar la conexión entre la representación gráfica de las palabras y el conocimiento lingüístico que posee y que le permite dotar de significado a la palabra escrita (modelo de doble vía, Jorm y Share, 1983) o modelos que explican su desarrollo (modelo de autoenseñanza, Share, 1995). Por este motivo y por razones de espacio, este artículo se centra fundamentalmente en los aspectos no específicos de la lengua escrita.

\section{Habilidades no específicas de la lectura}

En este apartado se analizan con más detalle las habilidades lingüísticas que son comunes a la comprensión de textos orales y escritos (véase parte izquierda de la Figura 2). Tomemos por ejemplo un título de prensa tal como: «Un hombre de color en la Casa Blanca: el sueño de Martin Luther King hecho realidad» (Alegría y Domín- 
guez, 2009). Para entender esta frase, tanto en forma escrita como oral, es necesario comprender las palabras que la componen, pero además hay que darles un sentido particular a las expresiones «hombre de color» $\mathrm{y}$ «Casa Blanca» que va más allá de las palabras que la componen, hay que tener conocimientos generales sobre el mundo, saber quién era Martin Luther King, etc. En la gran mayoría de los niños, el estar inmerso en un ambiente social que comunica oralmente suele ser suficiente para que tengan una base lingüística suficiente para comenzar el aprendizaje de la lectura. Estos niños normalmente poseen lo que podría llamarse una masa crítica de conocimientos lingüísticos y generales que reciben de su entorno (Rayner, Foorman, Perfett, Pesetsky y Seidenberg, 20oI). La lectura puede construirse sobre esta base. El corpus de conocimientos le permite al aprendiz lector comprender los textos que encuentra y esta misma actividad de lectura contribuye a ampliar estos conocimientos. Efectivamente es sobre todo en los textos donde el lector encuentra un vocabulario sofisticado y estructuras sintácticas ausentes o, al menos, poco frecuentes en la lengua oral, además de conocimientos e información. El Efecto Mateo, ampliamente desarrollado por Stanovich (1986), afirma que cuanto más sabe un lector, más aprende leyendo. Este efecto no se cumple en aquellos estudiantes que, por diferentes causas (dislexia, discapacidad auditiva, trastorno específico del lenguaje, entre otros), no poseen un bagaje lingüístico suficiente, impidiéndoles, por tanto, beneficiarse de los efectos positivos de la lectura sobre sus propios conocimientos lingüísticos y generales. Vamos a analizar con más detalle cada uno de estos tipos de conocimientos.

\section{I. Conocimientos lingüísticos}

\section{I.I. Vocabulario}

Tradicionalmente, se ha establecido una relación positiva entre la lectura y la adquisición de vocabulario, esto es, cuanto más lee una persona más acrecienta su léxico con palabras nuevas que, después, puede utilizar en sus intervenciones orales. De manera que, según esta afirmación, la importancia del vocabulario en la comprensión lectora aumenta con la edad y la experiencia con la lectura. Más recientemente, diversos autores (Mouzaki, Sideridis, Kotsolakou y Simos, 20I3; NICHD, 200o; Ouellette, 2006; Ouellette y Beers, 2010; Perfetti, 2007) señalan una relación en la dirección inversa, lo que supone que un buen nivel de vocabulario oral facilita el proceso lector y resulta fundamental en la comprensión de textos escritos. De hecho, el NICHD (2000) considera el vocabulario como uno de los componentes esenciales de la lectura y sostiene que la enseñanza explícita de vocabulario tiene efectos beneficiosos sobre la comprensión lectora.

Es preciso señalar que la mayoría de las investigaciones se centran en el estudio de la amplitud o vocabulario superficial, esto es, la cantidad de palabras que una persona conoce o, en términos de Ouellette (2006), el número de entradas léxicas; mientras que consideran en menor medida la profundidad de vocabulario o grado de representación semántica de estas. El conocimiento profundo de las palabras implica, al menos, las siguientes cuestiones: la relación de dicha palabra con ciertos temas o categorías semánticas; el acceso rápido al significado (o significados) y la capacidad de explicarlo y relacionarlo con el de otras palabras del mismo campo; y el empleo ade- 
MARCO TEÓRICO GENERAL PARA EVALUAR LA LECTURA

Y DISEÑAR PRÁCTICAS PARA SU ENSEÑANZA

ANA BELÉN DOMÍNGUEZ GUTIÉRREZ Y VIRGINIA GONZÁLEZ SANTAMARÍA

cuado de la palabra en diversas clases de construcciones. Estas podrían ser algunas estrategias que se deberían desarrollar en los alumnos cuando conocen ya una palabra (Ripoll y Aguado, 20I5).

Los resultados de las investigaciones muestran que el conocimiento profundo de vocabulario ejerce en la comprensión lectora una mayor influencia que el vocabulario superficial, es decir, que la calidad del conocimiento léxico predomina sobre la cantidad en la comprensión de textos escritos (Ouellette, 2006; Ouellette y Beers, 20IO; Perfetti, 2007). Ouellette y Beers (2010) comprobaron que, a medida que los niños crecen y se convierten en lectores cada vez más competentes, la decodificación pierde importancia, haciéndose progresivamente más relevantes los conocimientos de vocabulario oral y, esencialmente, de vocabulario profundo. Este hecho es acorde al planteamiento del Modelo Simple de Lectura, según el cual la relación de la comprensión lectora con la decodificación y la comprensión general de la lengua oral se ve modificada en función del nivel lector y la edad. Así, la decodificación es más importante al inicio del aprendizaje de la lectura invirtiéndose la relación en cursos superiores donde los procesos implicados en la decodificación ya deberían estar automatizados (Ripoll, Aguado y Castilla-Earls, 20I4). Por lo tanto, estos estudios subrayan la relevancia del vocabulario oral en la comprensión lectora y lo sitúan como un excelente predictor de la comprensión lectora.

De todo ello, puede deducirse una implicación para la práctica educativa de la enseñanza de la lectura: debería darse mayor importancia a la enseñanza de palabras nuevas, pero no teniendo en cuenta solamente la cantidad, sino haciendo un mayor hincapié en un conocimiento profundo de las mismas. Del mismo modo, debería realizarse una evaluación del vocabulario profundo y no solo superficial, como es lo habitual (generalmente a través del Peabody, Dunn, Dunn y Arribas, 2006). Para realizar una evaluación del vocabulario profundo contamos en nuestro país con la Prueba de Vocabulario de la Batería peale (Domínguez, Alegría, Carrillo y Soriano, 20I3, disponible en http://complydis.usal.es/).

\section{I.2. Conocimientos morfosintácticos}

En las dos últimas décadas se ha incrementado la investigación sobre la influencia de los conocimientos gramaticales, morfológicos y sintácticos en el aprendizaje de la lectura (Carlisle, 2000; Defior y Alegría, 2005; Pacton y Deacon, 2008). La investigación muestra la importancia del procesamiento de las estructuras morfosintácticas en el proceso de lectura y comprensión, así como en la explicación de dificultades de lectura de algunos estudiantes (Cain, 2oI4; Jiménez, Rodríguez, Guzmán y García, 20ıо; Nation y Snowling, 200o; Domínguez, Carrillo, González y Alegría, 20I6); ya que, para extraer el significado de un texto, no es suficiente con procesar las palabras aisladas, es necesario determinar cómo están relacionadas entre sí esas palabras. Para ello, es necesario tener presentes diversos indicadores, como los marcadores morfológicos o la propia estructura gramatical, es decir, el orden de las palabras en la oración y las distintas combinaciones de elementos (sintagmas y complementos) que pueden modificar el significado del mensaje y complejizar su comprensión.

La capacidad para utilizar las claves sintácticas se va perfeccionando a medida que el niño va desarrollando su lenguaje. Durante el desarrollo del lenguaje oral se van 
internalizando los patrones sintácticos en estrecha interdependencia con el desarrollo de otros componentes lingüísticos y en función de la experiencia y el contexto sociocultural de referencia. Sin embargo, hay algunas claves sintácticas que son específicas de la lectura y que los niños tienen que aprender para poder comprender los textos escritos. Tal y como señala Cuetos (1990), existen diferencias en el componente sintáctico entre la lengua oral y la escrita. En lengua oral hace falta relativamente poca sofisticación sintáctica para entender los mensajes porque, por una parte, están inmersos en un contexto y suelen ir acompañados de gestos, lo cual favorece la comprensión, $y$, por otra, porque en el lenguaje hablado los sonidos van acompañados de tonos del hablante (la prosodia) que no están presentes en la lectura. Además, los límites sintácticos están marcados por pausas que ayudan en el proceso de segmentación. El oyente ingresa las palabras que hay entre pausa y pausa en la memoria de corto plazo y las analiza como unidades de información antes de pasar al siguiente grupo de palabras. De hecho, cuando no existen pausas, la comprensión falla completamente. En cambio, en lengua escrita los límites sintácticos de las oraciones, frases y sintagmas vienen marcados por los puntos, las comas o simplemente se tienen que determinar a través de la propia estructura de la oración. El lector tiene, por tanto, que comprobar, con cada palabra que ingresa en la memoria de corto plazo, si completa una estructura constituyente (Kleiman, 1975). Si no es así, procede a ingresar la palabra siguiente. Tan pronto como considera que se ha completado una frase le extrae su significado, lo pasa a la memoria de largo plazo y deja libre la memoria de corto plazo para poder comenzar con el siguiente segmento de información.

Por ello, el funcionamiento de los procesos sintácticos podría ser un buen indicador para discriminar a personas con niveles funcionales de lectura de lectores con dificultades. En Educación Primaria, los alumnos con dificultades de lectura obtienen peores puntuaciones en tareas que requieren el manejo de claves sintácticas, tales como concordancias de género y número, interpretar el orden de las palabras en la oración, seleccionar la palabra funcional correcta para completar una oración o intuir dónde se deberían colocar signos de puntuación (Jiménez et al., 2010). De hecho, diversos trabajos muestran la existencia de correlación entre la competencia morfosintáctica de los alumnos de primaria y su comprensión lectora, indicando que la habilidad sintáctica es una variable muy predictiva de la comprensión lectora (Cain, Oakhill y Lemmon, 2004; Mokhtari y Niederhauser, 2013).

Además, hay muchos niños con trastornos en la lengua oral y/o discapacidad en los que la simple exposición a la lengua oral, en la interacción con personas de su entorno social, no es suficiente para desarrollar la morfosintaxis de su lengua. Estos niños pueden iniciar el aprendizaje de la lectura con un conocimiento de la gramática empobrecido (y del vocabulario). En este contexto, la pregunta que cabe hacerse es ¿qué hace un lector para comprender un texto que contiene palabras que no conoce y construcciones sintácticas que maneja «parcialmente»? Domínguez et al. (2016) han mostrado que estos lectores utilizan una estrategia que consiste en identificar las palabras clave de la frase, generalmente palabras frecuentes con contenido semántico pleno y elaboran sobre esta base un significado global. Los aspectos morfosintácticos de la frase se reducen a fórmulas elementales. Estos autores han denominado a esta estrategia Estrategia de Palabras Clave.

Para realizar una evaluación de la morfosintaxis contamos en nuestro país con las Pruebas de Morfología y de Sintaxis de la Batería peale y para la detección del uso de 
MARCO TEÓRICO GENERAL PARA EVALUAR LA LECTURA

Y DISEÑAR PRÁCTICAS PARA SU ENSEÑANZA

ANA BELÉN DOMÍNGUEZ GUTIÉRREZ Y VIRGINIA GONZÁLEZ SANTAMARÍA

la estrategia de palabras clave la prueba DEPC de esa batería (Domínguez et al., 20I3, véase en http://complydis.usal.es/).

Si a través de estas pruebas (u otras similares) se detecta en los estudiantes el uso de la Estrategia de Palabras Clave por problemas en la morfosintaxis, sería conveniente (o necesario) aplicar programas explícitos y sistemáticos de enseñanza de habilidades morfosintácticas, para que, aumentándolas, se reduzca el uso de la Estrategia de $\mathrm{Pa}$ labras Clave y, por consiguiente, mejoren los niveles lectores. Para desarrollar este trabajo puede ser útil el programa Las aventuras de Ana y Coco: en busca del cromo perdido, que también puede encontrarse gratuitamente en https://complydis.usal.es/.

Por otro lado, es preciso señalar que no todas las dificultades de comprensión de estructuras sintácticas son debidas a trastornos de lenguaje. Incluso los lectores hábiles pueden tener dificultades en la comprensión de oraciones muy largas, oraciones con estructuras complejas, oraciones con significado ambiguo y con expresiones poco frecuentes como «mas» (sin tilde), «en cuyo caso» o «no ha lugar a» (Ripoll y Aguado, 20I5). Sánchez, García y Bustos (2010) propusieron la existencia de una competencia retórica receptiva (véase más adelante) o habilidad para manejar los recursos del discurso que permiten interpretar el texto, entre ellos las palabras funcionales, el orden de las palabras, las elipsis y las sustituciones. Podría haber dificultades de comprensión debidas a que los recursos que se utilizan en los textos escritos pueden ser más sofisticados que los que se emplean en la lengua oral. No es frecuente escuchar expresiones como «sin que obedezca a las causas expuestas», si no es en algún discurso académico, de modo que cuando alguien se encuentra ante este tipo de construcciones en el texto escrito puede tener dificultades para interpretarlas. Según los resultados de esos autores, la habilidad que tienen los alumnos de $6{ }^{\circ}$ de primaria para interpretar referencias, como saber de qué o quién habla el texto cuando dice "este explorador» o «esta desgracia», y la sensibilidad para interpretar expresiones como «en primer lugar», «por lo que» o «algunos» explican una parte de la comprensión lectora de esos alumnos de la que no dan cuenta los conocimientos sobre el tema, la precisión de la lectura ni la memoria de trabajo.

Finalmente, es importante señalar que, aunque es posible acceder conscientemente a nuestros conocimientos sobre estructuras gramaticales y ejercer un control intencional en relación con ellas, la mayor parte del procesamiento morfosintáctico de la lengua escrita lo efectuamos de modo automatizado, lo que contribuye significativamente a liberar recursos que permitan abordar actividades complejas relacionadas con la integración textual y la comprensión. Asunto que se aborda en el punto siguiente.

\subsection{Conocimientos previos (generales, tema o disciplina)}

Comprender un texto es construir una representación mental coherente, para lo cual hay que ejecutar determinados procesos mentales, que se apoyan en ciertos conocimientos (García, Sánchez y García, 20I4). Para comprender es necesario integrar las ideas del texto entre sí, pero también integrar esos contenidos con nuestros conocimientos, esto es, la comprensión de un texto supone una conexión constante entre el texto y lo que ya sabemos. Además de los conocimientos sobre el lenguaje descritos anteriormente, el acceso a los conocimientos previos es una actividad constante a lo largo del proceso de comprensión: debemos acceder al significado de las palabras, 
debemos acceder a nuestros conocimientos sobre el mundo para conectar causalmente o temáticamente unas proposiciones a conocimientos específicos sobre un tema o disciplina o relacionados con el tópico tratado en el texto, lo que nos permite anticipar ciertas categorías de información, y a conocimientos específicos sobre cómo acceder a múltiples textos o documentos, como es habitual en el caso de la lectura digital.

El acceso a estos conocimientos previos permite realizar inferencias o aportar al proceso de comprensión elementos que el texto en sí no proporciona directamente. Es decir, durante los procesos de comprensión, el lector no debe limitarse a decodificar y formar ideas con la información presente en el texto, para crear en su mente una representación igual que la que tenía la persona que lo escribió (comprensión superficial), sino que debe aportar información a partir de sus conocimientos y llevar a cabo procesos de integración entre el texto y lo que sabe (comprensión profunda).

La construcción de inferencias no es algo exclusivo de la lectura, probablemente es una propiedad general del pensamiento humano que se manifiesta en distintas formas de comprensión, de modo que también se construyen inferencias al interpretar la lengua oral, al ver una película o al comprender una imagen (Ripoll y Aguado, 20I5). Estos autores señalan que la habilidad para construir inferencias se desarrolla a una edad muy temprana, antes de que los niños hayan aprendido a leer. A los 4 años los niños muestran claramente su habilidad para construir inferencias al escuchar historias o verlas en televisión; y, además, se trata de la misma habilidad que van a emplear posteriormente en la comprensión de textos.

Y, como concluyen García et al. (20I4), si aún cabe alguna duda de que los conocimientos previos son cruciales, merece la pena destacar que también predicen la comprensión de un texto, esto es, a más conocimientos más comprensión. En definitiva, sin conocimientos previos no es posible decodificar, formar ideas o hacer inferencias.

\subsection{Conocimientos formales (estructuras retóricas y organizativas de los textos)}

Leer con fluidez las palabras de un texto permite liberar recursos de atención y memoria de trabajo, lo que posibilita que se dediquen a la consecución de una lectura eficiente. Existen lectores que alcanzan esta habilidad en los tres primeros cursos de la Educación Primaria. Pero esto no es suficiente para muchos estudiantes, generalmente a partir de cuarto o quinto de Primaria, cuando se enfrentan a textos académicos, comienzan a experimentar dificultades que hasta ese momento no se producían. Además, deben ir adquiriendo la capacidad de generar sus propios objetivos de lectura y regular desde ellos la lectura, capacidad que es más tardía y que un amplio número de alumnos no adquiere (Sánchez et al., 20Io). Es decir, como señalan estos autores, los alumnos deben operar con textos académicos que están organizados retóricamente mediante señalizadores (como «por un lado», «en resumen» o «más importante todavía») que sirven de guía durante la lectura. El dominio de estos recursos y estructuras retóricas enriquece las competencias orales de partida. Lo que conforma el lenguaje académico. Y, además, deben generar metas específicas para la lectura de un texto y revisar en qué grado esas metas se van consiguiendo, lo que puede llevar o no a desplegar ciertas acciones reparadoras a las que se denomina estrategias. En esto consiste la lectura estratégica. A estas acciones mentales: establecer un objetivo (para qué leer), detectar problemas (evaluar si el objetivo se va cumpliendo, detectando problemas 
MARCO TEÓRICO GENERAL PARA EVALUAR LA LECTURA

Y DISEÑAR PRÁCTICAS PARA SU ENSEÑANZA

ANA BELÉN DOMÍNGUEZ GUTIÉRREZ Y VIRGINIA GONZÁLEZ SANTAMARÍA

que puedan surgir) y reparar esos problemas mediante estrategias (como p. e., releer ciertos segmentos del texto...), se las denomina procesos de autorregulación (véase parte inferior derecha de la Figura 2).

En palabras de Sánchez et al. (20IO), «esto quiere decir que la lectura proporciona experiencias comunicativas que no son habituales en la vida cotidiana, que es donde se fraguan inicialmente las competencias lingüísticas orales. Por ello, al entrar en contacto con los textos académicos, los alumnos tienen que aprender a detectar, utilizar y usar marcas retóricas ( «hay que encontrar tres características», «hay que rellenar esta laguna...»). Y, además, esas nuevas competencias se pueden extender a situaciones comunicativas orales, lo que permite a los alumnos exitosos seguir un debate de cierta altura («hay tres objeciones en las que deseo detenerme»...), entender una explicación compleja o participar ellos mismos en una discusión grupal sobre algún tema. Los estudiantes tienen que volverse sensibles a las señalizaciones mencionadas («una segunda razón es...») y aprender a operar conscientemente con ellas («Si leo una segunda razón, eso quiere decir que ya se ha expuesto una primera razón y que quizás vaya a haber una tercera...»). El conocimiento y uso del repertorio de señales que pueden ser insertadas en un texto complejo y que, llegado el caso, pueden facilitar el despliegue de estrategias es lo que se denomina competencia retórica (Sánchez et al., 20Io). Los trabajos de estos autores muestran que el dominio de estas estructuras no es inmediato. Los marcadores de la estructura global del texto («en primer lugar», «otra razón») suelen pasar desapercibidos a los estudiantes de $4 .^{\circ}$ curso de Primaria y a los alumnos de bajo nivel de $6 .^{\circ}$. Recursos aún más sofisticados como «es sabido que... pero...» son adquiridos aún más tardíamente. Y expresiones como «este fenómeno» resultan costosas de manejar en los niveles educativos intermedios ( $4 .^{\circ}-6 .^{\circ}$ de Primaria).

En otras palabras, además de lo expuesto, los textos incluyen marcadores discursivos de distintos tipos que actúan como instrucciones potenciales para aclarar el tratamiento que debe darse a esas ideas (Sánchez et al., 20Io). Según estos autores, conocer, detectar y dejarse llevar por estos marcadores constituye la competencia retórica. Señalan, también, que hay un amplio número de marcadores discursivos muy distintos, pero que pueden ser agrupados en función del tipo de procesos de lectura que facilitan. Así, se pueden encontrar marcadores que sostienen el procesamiento local o el global, pero también marcadores que promueven los procesos de integración texto-conocimientos y de autorregulación.

\section{Conclusiones}

El marco teórico expuesto permite identificar los conocimientos y procesos que dan lugar a la comprensión lectora y que pueden ser evaluados, enseñados y mejorados, es decir, es una guía para diseñar prácticas de enseñanza de la lengua escrita. En este diseño, además de abordar los procesos de reconocimiento de las palabras escritas, se deberían plantear también lo que hemos denominado procesos no específicos de la lectura, esto es, los conocimientos lingüísticos del lector, esencialmente las competencias léxicas (es decir, el conocimiento del sentido de las palabras, así como procesos de análisis sintáctico y de integración semántica), además de los conocimientos generales sobre el mundo y de los temas en particular; y los conocimientos formales de los textos. 
De forma más concreta, el diseño de prácticas de enseñanza de la lectura se podría plantear en función de esos dos grupos de factores o habilidades, enseñando de forma explícita y sistemática estrategias y/o procesos:

Procesos de reconocimiento de la palabra escrita:

- Desarrollar conciencia fonológica.

- Adquirir el sistema de reglas de correspondencia grafema-fonema.

- Automatizar las reglas de correspondencia grafema-fonema.

- Aprender a leer de forma directa grupos de letras e incluso palabras completas.

Estrategias de comprensión:

- Favorecer el lenguaje escrito desde el lenguaje oral (conocimientos lingüísticos: léxico-semántico, morfosintáctico y pragmático).

- Activar los conocimientos previos (generales sobre el mundo, específicos de los temas o disciplinas).

- Detectar y utilizar conocimientos formales (organizativos de los textos y estructuras retóricas).

- Hacer explícitas las estrategias que un lector experto pone en marcha cuando lee o escribe antes, durante y al final del proceso.

Finalmente, señalar que en el proceso de intervención es necesaria la contextualización de las actividades que se van a desarrollar, esto es, crear contextos de lectura y escritura que den sentido a ese modo estratégico (contextos de aprendizaje significativos y comunicativos). Es importante y necesario partir de lo que en cada momento estén trabajando los alumnos en sus aulas, teniendo siempre presente el tipo de metodología y actividades que en las mismas se estén desarrollando (p. e., Proyectos de Trabajo; Unidad Didáctica; Proyecto comunicativo; o sobre personajes de cuentos; o acerca de las partes del cuerpo...). Es decir, no se trata de introducir un programa o actividades metafonológicas, metalingüísticas o de comprensión del lenguaje en el aula de forma paralela a lo que se realiza en los bloques de contenidos curriculares, sino, más bien, incluir estas tareas en esos bloques.

\section{Bibliografía}

Alegría, J. (2004). Deafness and Reading. En T. Nunes y P. Bryant (eds.), Handbook of Children's Literacy (pp. 459-489). Dordrecht: Kluwer Academic Publishers.

Alegría, J. (2006). Por un enfoque psicolingüístico del aprendizaje de la lectura y sus dificultades -20 años después-. Infancia y Aprendizaje, 29(I), 93-III. doi:Io.II74/021037006775380957

Alegría, J. y Domínguez, A. B. (2009). Los alumnos sordos y el aprendizaje de la lectura. Revista Latinoamericana de Educación Inclusiva, 3(I), 95-III. http://www.rinace.net/rlei/ numeros/vol3-numi/

AA. VV. (2012). La atención al alumnado con dislexia en el sistema educativo en el contexto de las necesidades especificas de apoyo educativo. Madrid: MECD-Centro Nacional de Innovación e Investigación Educativa. Colección Eurydice REDIE.

CaIN, K. (20I4). Reading development and difficulties. Oxford: Wiley-Blackwell. 
MARCO TEÓRICO GENERAL PARA EVALUAR LA LECTURA

Y DISEÑAR PRÁCTICAS PARA SU ENSEÑANZA

ANA BELÉN DOMÍNGUEZ GUTIÉRREZ Y VIRGINIA GONZÁLEZ SANTAMARÍA

Cain, K.; OAkhill, J. y Lemmon, K. (2004). Individual Differences in the Inference of Word Meanings From Context: The Influence of Reading Comprehension, Vocabulary Knowledge, and Memory Capacity. Journal of Educational Psychology, 96, 67I-68I. doi: I0.1037/0022-0663.96.4.67I

Carlisle, J. F. (2000). Awareness of the structure and meaning of morphologically complex words: Impact on reading. Reading \& Writing, I2(3), I69-I90. doi: I0.I023/A:I008131926604

Cuetos, F. (1990). Psicología de la lectura. Madrid: Editorial Escuela Española.

Defior, S. y Alegría, J. (2005). Conexión entre morfosintaxis y escritura: cuando la fonología es (casi) suficiente para escribir. Revista de Logopedia, Foniatría y Audiología, 25, 5I-6I. doi: I0.IOI6/So2I4-4603(05)75816-2

Defior, S. y Serrano, F. (20iI). Procesos Fonológicos Explícitos e Implícitos, Lectura y Dislexia. Revista Neuropsicología, Neuropsiquiatría y Neurociencias, II(I), 79-94.

Domínguez, A. B. (2009). Fonología sin audición. Aula. Revista de Pedagogía de la Universidad de Salamanca, I5, I39-I53.

Domínguez, A. B.; Alegría, J.; Carrillo, M. y Soriano, J. (20I3). Peale. Pruebas de Evaluación Analítica de Lengua Escrita. Universidad de Salamanca. Número de asiento registral: oo/2013/4067. https://complydis.usal.es/

Domínguez, A. B.; Carrillo, M. S.; González, V. y Alegría, J. (2016). How do deaf children with and without cochlear implants manage to read sentences: The key word strategy. Journal of Deaf Studies and Dead Education, 2I(3), 280-292. doi: I0.1093/deafed/enwo26

Domínguez, A. B. y Leybaert, J. (20I4). Acceso a la estructura fonológica de la lengua: repercusión en los lectores sordos. Aula. Revista de Pedagogía de la Universidad de Salamanca, 20, II época, 65-8I. ISSN 2174-0925.

Dunn, L. M.; Dunn, L. M. y Arribas, D. (2006). PPVT-III PeAbody: Test de vocabulario en imágenes: manual. Madrid: TEA Ediciones.

García, H.; Sánchez, E. y García, R. (20I4). Comprensión de textos. Dificultades, evaluación y ayudas. En J. N. García SÁnchez (coord.), Prevención en dificultades del desarrollo $y$ del aprendizaje. Madrid: Pirámide.

Gough, P. B. y Tunmer, W. E. (1986). Decoding, reading, and reading disability. Remedial and Special Education, 7(I), 6-Io. doi: 10.1177/074193258600700104

Jiménez, J. E.; Rodríguez, C.; Guzmán, R. y García, E. (20Io). Desarrollo de los procesos cognitivos de la lectura en alumnos normolectores y alumnos con dificultades específicas de aprendizaje. Revista de Educación, 353, 36I-386.

Jorm, A. y Share, D. (1983). Phonological recoding and reding acquisition. Applied Psycholinguistics, 4, I03-I47.

KLeiman, G. M. (1975). Speech recoding in reading. Journal of Verbal Learning \& Verbal Behavior, I4(4), 323-339. doi: 10.1016/Soo22-5371(75)80013-2

Mokhtari, K. y Niederhauser, D. S. (20I3). Vocabulary and Syntactic Knowledge Factors in sth Grade Students' Reading Comprehension. International Electronic Journal of Elementary Education, 5(2), 157-170.

Morais, J. (1994). El arte de leer. Madrid: Aprendizaje-Visor.

Mouzaki, A.; Sideridis, G. D.; Kotsolakou, A. y Simos, P. G. (20I3). The Role of Vocabulary in the Context of the Simple View of Reading. Reading E Writing Quarterly. Overcoming Learning Difficulties, 29, I68-202. doi: 10.1080/10573569.2013.758569

Nation, K. y SNOwling, M. J. (2000). Factors influencing syntactic awareness skills in normal readers and poor comprehends. Applied Psycholinguistics, 2I(2), 229-24I. doi: Io.IOI7/ Sor 42716400002046

NichD. National Institute of Child Health and Human Development (2000). Report of the National Reading Panel. Teaching children to read: An evidence-based assessment of the scientific research literature on reading and its implications for reading instruction (NIH $\mathrm{Pu}-$ blication No. oo-4769). Washington, DC: U.S. Government Printing Office. 
OAKhill, J.; Cain, K. y Bryant, P. E. (2003). The dissociation of word reading and text comprehension: Evidence from component skills. Language and Cognitive Processes, $18(4), 443-$ 468. doi: I0.1080/01690960344000008

Ouellette, G. P. (2006). What's Meaning Got to Do With It: The Role of Vocabulary in Word Reading and Reading Comprehension. Journal of Education Psychology, 98(3), 554566. doi:10.1037/0022-0663.98.3.554

Ouellette, G. P. y Beers, A. (20I0). A not-so-simple view of reading: how oral vocabulary and visual-word recognition complicate the story. Reading $\mathcal{E}$ Writing, 23(2), 189-208. doi: I0.1007/SIII45-008-9159-I

Pacton, S. y Deacon, S. H. (2008). The timing and mechanisms of children's use of morphological information in spelling: A review of evidence from English and French. Cognitive Development, 23(3), 339-359. doi: 10.1016/j.cogdev.2007.09.004

Perfetti, C. (2007). Reading Ability: Lexical Quality to Comprehension. Scientific Studies of Reading, II(4), 357-383. doi: I0.1080/1088843070I530730

Rayner, K.; Foorman, B. R.; Perfetti, C. A.; Pesetsky, D. y Seidenberg, M. S. (200i). How psychological science informs the teaching of reading. Psychological Science in the Public Interest, 2, 3I-74. doi: 10.IIII/1529-1006.00004

Ripoll, J. C. y Aguado, G. (20I5). Enseñar a leer. Cómo hacer lectores competentes. Madrid: Editorial EOS.

Ripoll, J. C.; Aguado, G. y Castilla-Earls, A. P. (20I4). The simple view of reading in elementary school: A systematic review. Revista de Logopedia, Foniatría y Audiología, 34(I), I7-3I. doi: I0.IOI6/j.rlfa.2013.04.006

Sánchez, E.; García, R. y Bustos, A. (20Io). La comprensión oral y escrita: ¿es la decodificación de las palabras la única diferencia? En M. CARrillo y A. B. Domínguez (coords.), Dislexia y sordera. Lineas actuales en el estudio de la lengua escrita y sus dificultades (pp. 37-60). Málaga: Aljibe.

Savage, R. (200I). The «Simple View» of Reading: Some evidence and possible implications. Educational Psychology in Practice, I7(I), 17-33. doi: I0.1080/02667360120039951

Share, D. L. (1995). Phonological recoding and self-teaching: Sine qua non of reading acquisition. Cognition, 55, I5I-218. doi: 10.1016/0010-0277(94)00645-2

STANovich, K. E. (I986). Matthew effects in reading: Some consequences of individual differences in the acquisition of literacy. Reading Research Quarterly, 2I, 360-407. doi: I0.1598/ RRQ.2I.4.I

Wagner, R. K. y Torgesen, J. K. (1987). The nature of phonological processing and its causal role in the acquisition of reading skills. Psychological Bulletin, Ior(2), 192-212. doi: I0.1037/0033-2909.IOI.2.192 